\title{
SOME GLOBAL RESULTS ON EXTENSION OF CR-OBJECTS IN COMPLEX MANIFOLDS
}

\author{
GUIDO LUPACCIOLU
}

\begin{abstract}
We prove some results concerning the holomorphic extendability of CR-objects defined on real hypersurfaces of a complex manifold. After a preliminary generalization of the classic theorem on the extendability from the boundary of a relatively compact domain, we discuss the extendability from a part of the boundary of such a domain, the one side extendability from a piece of hypersurface and the extendability from the boundary of an unbounded domain.
\end{abstract}

Let $M$ be a noncompact connected complex-analytic manifold with countable topology, of complex dimension $n \geq 2$.

The present paper deals with holomorphic extension of CR-objects defined on real hypersurfaces of $M$. What in fact we do for the most part is to discuss some generalizations in different directions of the well-known global extension theorem which asserts the existence and uniqueness of a holomorphic extension on an open domain $D \Subset M$ for a CR-function defined on the boundary $b D$ of $D$, under the condition $H_{c}^{1}(M, \mathscr{O})=0$ (cf. [9]).

The CR-objects considered here are CR-sections, CR-distribution sections and CR-hyperfunction sections of a holomorphic complex vector bundle on $M$.

Our first result (Theorem 1.1) relates to the holomorphic extension of these kinds of CR-objects from the boundary $b D$ of a domain $D \subset M$ which need not be relatively compact, but the closure of which is supposed to belong to some paracompactifying family in $M$ (not the whole family of closed sets). Such a generalization of the global extension theorem does not seem to be already found in the literature; actually, although it does not require any essentially new ideas, is worth being discussed, as it allows one to derive other results of some independent interest on extension of CR-objects.

Later, in $\S 2$, we discuss the question of holomorphic extendability on all of a domain $D \Subset M$ for CR-objects which need not be defined on the whole boundary $b D$, but just on the complement $b D \backslash K$ of a closed set $K \subset b D$. This question has already been investigated, in more particular settings (cf. [11, $13,14,16,17,22,23])$, by using integral representations techniques. Here we

Received by the editors September 10, 1987 and, in revised form, March 3, 1988 and November 15,1988 .

1980 Mathematics Subject Classification (1985 Revision). Primary 32D99. 
generalize most of the results obtained so far by resorting to the theorem of $\S 1$ and using sheaf-theoretic techniques.

Next, in $\S 3$, we derive from the previous discussion some results concerning the one side extension property for the CR-objects defined on a piece of a real hypersurface in $\mathbf{C}^{n}(n \geq 2)$. In particular we derive a proposition (Corollary 3.2) which implies both the global and the local classical extension theorems.

Finally, in $\S 4$, we prove a theorem which gives a sufficient condition for an unbounded domain $D \subset \mathbf{C}^{n} \quad(n \geq 2)$ under which the CR-objects defined on $b D$ can be extended holomorphically on $D$.

\section{Global EXTENSION FROM THE WHOLE BOUNDARY}

Let $D$ be an open domain in $M$, not necessarily with compact closure, with smooth boundary $b D$ of class $C^{v}, 1 \leq v \leq \infty$, or of class $C^{\omega}$ (i.e. real-analytic).

Let $W$ be a holomorphic complex vector bundle on $M$ of finite rank $r \geq 1$.

If $u$ is an integer with $0 \leq u \leq v$, we let $C^{u}(b D, W)$ and $C^{u}(\bar{D}, W)$ be the spaces of sections of class $C^{u}$ of $W$ on $b D$ and $\bar{D}$; if $b D$ is of class $C^{\infty}$, we let $\mathscr{D}^{\prime}(b D, W)$ be the space of distribution sections of $W$ on $b D$; moreover, if $b D$ is of class $C^{\omega}$, we let $\mathscr{B}(b D, W)$ be the space of hyperfunction sections of $W$ on $b D$.

Given an open set $U \subset M$ such that $\left.W\right|_{U}$ is trivial and $b D \cap U \neq \varnothing$, an element $f$ of $C^{u}(b D, W)$, or $\mathscr{D}^{\prime}(b D, W)$, or $\mathscr{B}(b D, W)$ can be expressed on $b D \cap U$ in terms of a local base $\left(s_{1}, \ldots, s_{r}\right)$ of $\Gamma(U, \mathscr{O}(W))$ as

$$
f=\sum_{j=1}^{r} f_{j} \otimes s_{j}
$$

with the $f_{j}$ 's functions of class $C^{u}$, or distributions, or hyperfunctions, respectively, on $b D \cap U$.

Let us say that $f$ is a $C R$-object on $b D$ with coefficients in $W$, and let us write

$$
f \in \mathbf{C R}(b D, W),
$$

in case, for every $U$ and $\left(s_{1}, \ldots, s_{r}\right)$ as above, the $f_{j}$ 's are CR-functions, or CR-distributions, or CR-hyperfunctions. We refer to [9,12 and 18] for basic information.

It is known (cf. the same references) that, when $\bar{D}$ is compact and $b D$ is connected, every $f$ in $\operatorname{CR}(b D, \mathbf{1})$, or in $\operatorname{CR}\left(b D, \Lambda^{p} H^{*}(M)\right),{ }^{1}$ is the boundary value, in the appropriate sense, of a unique holomorphic function $F \in \mathscr{O}(D)$, or form $F \in \Omega^{p}(D)$, provided $H_{c}^{1}(M, \mathscr{O})=0$, or $H_{c}^{1}\left(M, \Omega^{p}\right)=0$, respectively. In particular, if $f$ is in $C^{u}(b D, \mathbf{1})$, or in $C^{u}\left(b D, \Lambda^{p} H^{*}(M)\right)$, then $F$ has an extension of class $C^{u}$ to $\bar{D}$, which is equal to $f$ on $b D$.

\footnotetext{
${ }^{1}$ Here 1 means the trivial line bundle on $M$ and $H^{*}(M)$ the holomorphic co-tangent bundle of $M$.
} 
The starting point of this paper is the following generalization of the above results.

Theorem 1.1. Let $\Phi$ be a paracompactifying family in $M$, not the one of all closed sets, and suppose that

$$
H_{\Phi}^{1}(M, \mathscr{O}(W))=0 .
$$

Then, if $\bar{D} \in \Phi$ and $b D$ is connected, every CR-object $f \in \mathrm{CR}(b D, W)$ is the boundary values of a unique holomorphic section $F \in \Gamma(D, \mathscr{O}(W))$.

Moreover, if $f \in C^{u}(b D, W)$, then $F \in C^{u}(\bar{D}, W)$ and $\left.F\right|_{b D}=f$.

Let us recall that a family $\Phi$ of closed subsets of $M$ is said to be a paracompactifying family in $M$ if every closed subset of a member of $\Phi$ and every finite union of members of $\Phi$ are themselves members of $\Phi$, moreover every member of $\Phi$ has some neighborhoods which are members of $\Phi$ as well.

It is plain that for $\Phi=c$, the family of all compact subsets of $M$, Theorem 1.1 yields again the recalled known results (aside from the more general coefficients).

The proof of Theorem 1.1 can be developed along the same lines as in [9] for the case $f \in C^{u}(b D, W)$ as in [12] for the case $f \in \mathscr{D}^{\prime}(b D, W)$ and as in [18] for the case $f \in \mathscr{B}(b D, W)$. Indeed, the replacement of $c$ by $\Phi$ and the consideration of more general coefficients do not require any essentially new arguments.

In the smooth case one can also adapt the simple argument used in the classical book by Hörmander [10, Theorem 2.3.2'], as outlined below.

One first extends $f$ to a smooth section $\tilde{f}$ of $W$ on $\bar{D}$, such that $\bar{\partial} \tilde{f}=0$ on $b D$, and then solves the equation $\bar{\partial} v=\alpha \quad(\alpha=\bar{\partial} \tilde{f}$ on $D$, and $\alpha=0$ on $M \backslash D$ ) by a global smooth section $v$ of $W$ supported in $\Phi$ (which is possible by the conditions $\bar{D} \in \Phi$ and $\left.H_{\Phi}^{1}(M, \mathscr{O}(W))=0\right)$; since $v$ is holomorphic on $M \backslash \bar{D}, M \backslash \bar{D}$ is connected and $(M \backslash \bar{D}) \backslash \operatorname{supp}(v) \neq \varnothing$ (because $\Phi$ is not the whole family of closed sets), it follows that $v=0$ on $M \backslash D$, and then $F=\tilde{f}-v$ is the asserted extension of $f$.

\section{GLOBAL EXTENSION FROM A PART OF THE BOUNDARY}

Throughout this section $D$ will denote a relatively compact open domain in $M$ and $K$ a closed subset of $b D$, in such a way that $b D \backslash K$ is connected and smooth of class $C^{v}, 1 \leq v \leq \infty$, or of class $C^{\omega}$.

No regularity assumption is made on $K$.

We shall be concerned with the question of holomorphic extendability on the whole $D$ of the CR-objects with coefficients in $W$ defined on the relatively open part $b D \backslash K$ of $b D$. We let $\operatorname{CR}(b D \backslash K, W)$ denote the set of these CRobjects, of the various kinds specified in the previous section, according to the regularity of $b D \backslash K$.

For the sake of conciseness in statements, we shall simply say that the global extension property holds for $\operatorname{CR}(b D \backslash K, W)$, in order to mean the following: 
Every $f \in \mathrm{CR}(b D \backslash K, W)$ is the boundary values on $b D \backslash K$, in the appropriate sense, of a unique holomorphic section $F \in \Gamma(D, \mathscr{O}(W))$; moreover, if $f \in$ $C^{u}(b D \backslash K, W), 0 \leq u \leq v$, then $F$ admits an extension of class $C^{u}$ to $\bar{D} \backslash K$ which coincides with $f$ on $b D \backslash K$.

The following theorem is the basic result of this section.

Theorem 2.1. Suppose that $H_{c}^{1}(M, \mathscr{O}(W))=0$ and that there exists a compact set $E \subset M$ verifying the following conditions:

(i) $E \cap \bar{D}=K$;

(ii) $M \backslash E$ is connected;

(iii) The homomorphism induced by inclusion

$$
\underset{\lim }{\longleftarrow} H_{c}^{2}(U, \mathscr{O}(W)) \rightarrow H_{c}^{2}(M, \mathscr{O}(W))
$$

( $U$ open, $U \supset E$ ) is injective.

Then the global extension property holds for $\mathrm{CR}(b D \backslash K, W)$.

Proof. Let $\mathscr{U}$ be a neighborhood basis of $E$ of relatively compact open sets such that, for each $U \in \mathscr{U}, M \backslash U$ is connected. Then, since $H_{c}^{1}(M, \mathscr{O}(W))=$ 0 , the restrictions $H^{0}(M, \mathscr{O}(W)) \rightarrow H^{0}(M \backslash U, \mathscr{O}(W)), U \in \mathscr{U}$, are isomorphisms (Hartogs's theorem). This implies (cf. [3, Lemma 2.13]) that the homomorphism

$$
H^{1}(M \backslash E, \mathscr{O}(W)) \rightarrow \lim _{U \in \mathscr{U}} H^{1}(M \backslash U, \mathscr{O}(W))
$$

given by restriction is an isomorphism too. It follows (by the same reasoning as in [3, p. 32]) that the canonical homomorphism

$$
H_{E}^{2}(M, \mathscr{O}(W)) \rightarrow \lim _{\longleftarrow} H_{c}^{2}(U, \mathscr{O}(W))
$$

is injective. Therefore, by condition (iii), the homomorphism $H_{E}^{2}(M, \mathscr{O}(W))$ $\rightarrow H_{c}^{2}(M, \mathscr{O}(W))$ induced by inclusion is injective too.

Now, let $M^{\prime}=M \backslash E$ and $\Phi=c \cap M^{\prime}$ be the family of all the intersections of compact subsets of $M$ with $M^{\prime}$, i.e. of all the closed subsets of $M^{\prime}$ which are relatively compact in $M$. Clearly $\Phi$ is a paracompactifying family in $M^{\prime}$, and the exact cohomology sequence

$$
\begin{aligned}
\cdots \rightarrow H_{E}^{q}(M, \mathscr{O}(W)) & \rightarrow H_{c}^{q}(M, \mathscr{O}(W)) \\
& \rightarrow H_{\Phi}^{q}\left(M^{\prime}, \mathscr{O}(W)\right) \rightarrow H_{E}^{q+1}(M, \mathscr{O}(W)) \rightarrow \cdots
\end{aligned}
$$

gives

$$
H_{\Phi}^{1}\left(M^{\prime}, \mathscr{O}(W)\right)=0 .
$$

Then, since $D \subset M^{\prime}, \operatorname{Clos}_{M^{\prime}}(D)=\bar{D} \backslash K \in \Phi, \operatorname{Bd}_{M^{\prime}}(D)=b D \backslash K$, and, moreover, $M^{\prime}$ is connected and $\Phi$ is not the whole family of closed subsets of $M^{\prime}$, the conclusion follows from Theorem 1.1. Q.E.D. 
Remark. Condition (iii) of Theorem 2.1 is verified in particular if there is a basis $\mathscr{U}$ of open neighborhoods of $E$ such that the homomorphisms $H_{c}^{2}(U, \mathscr{O}(W))$ $\rightarrow H_{c}^{2}(M, \mathscr{O}(W)), U \in \mathscr{U}$, induced by inclusion are injective. This, translated in terms of Dolbeault cohomology, means the following:

Let $g$ be a smooth $W$-valued $\bar{\partial}$-closed $(0,2)$-form on $M$ with compact support in a small neighborhood of $E$. Then, if the

(*) equation $\bar{\partial} u=g$ can be solved with compact support in $M$, it can also be solved with compact support in a small neighborhood of $E$.

In the smooth case there is a simple direct proof for the version of Theorem 2.1 in which $E$ verifies the above condition, by the following further adaptation of Hörmander's argument [10, Theorem 2.3.2'].

The form $\alpha$ (see the end of the previous section) is now defined only on $M \backslash E$ and is 0 outside $D$. Pick a cutoff function $\chi, \chi=0$ on $E$ and $\chi=1$ outside a small neighborhood of $E$, and consider the $(0,2)$-form $g=\bar{\partial}(\chi \alpha)$. Due to condition $(*)$, we can find a $(0,1)$-form $k$, supported near $E$, such that $\bar{\partial} k=$ $g$. Set $\beta=\chi \alpha-k$. Then $\beta$ coincides with $\alpha$ outside a small neighborhood of $E$ and provides us with a $\bar{\partial}$-closed extension of $\alpha$ to the whole $M$. Due to the condition $H_{c}^{1}(M, \mathscr{O}(W))=0$, we can find a compactly supported global section $v$ of $W$ with $\bar{\partial} v=\beta$. Then $F=\tilde{f}-v$ is a holomorphic extension of $f$ to the complement in $D$ of a small neighborhood of $E$, and the proof ends by shrinking this neighborhood.

The main applications of Theorem 2.1 are probably those relating to the case where the manifold $M$ is $(n-2)$-complete, since $(n-2)$-completeness seems to be the most general quite verifiable condition which guarantees the vanishing of $H_{c}^{1}(M, \mathscr{O}(W))$, for any $W$.

Let us recall that $M$ is called $q$-complete $(0 \leq q \leq n)$ in case there is an exhaustion function of class $C^{\infty}$ on $M$ which is a strongly $q$-plurisubharmonic, i.e. whose Levi form admits everywhere at least $n-q$ positive eigenvalues. Andreotti and Grauert [1] proved that, if $M$ is $q$-complete, $H^{j}(M, \mathscr{F})=0$ when $j>q$ for every coherent analytic sheaf $\mathscr{F}$ on $M$.

Hence, if our manifold $M$ is $(n-2)$-complete, it follows, via the Serre duality theorem, that $H_{c}^{1}(M, \mathscr{O}(W))=0$, as asserted above.

Now let us give the following

Definition. A compact set $E \subset M$ is called q-complete $(0 \leq q \leq n)$ in case it admits a neighborhood basis $\mathscr{U}$ of relatively compact q-complete open sets.

It is important for the following to point out that, if $E$ is $(n-2)$-complete, then $M \backslash E$ is connected.

In fact we have $H^{2 n-1}(E, \mathbf{R}) \cong \lim _{U \in \mathscr{U}} H^{2 n-1}(U, \mathbf{R})$, and it is known that, due to $(n-2)$-completeness, $H^{2 n-1}(U, \mathbf{R})=0$ for each $U \in \mathscr{U}$. Hence $H^{2 n-1}(E, \mathbf{R})=0$, which implies the connectedness of $M \backslash E$. 
That being stated, a first obvious consequence of Theorem 2.1 is

Corollary 2.2. Suppose that $n \geq 3, M$ is $(n-2)$-complete and there exists an $(n-3)$-complete compact set $E \subset M$ with $E \cap \bar{D}=K$. Then, for any $W$, the global extension property holds for $\mathrm{CR}(b D \backslash K, W)$.

We wish to observe that, if in the above corollary $M$ is assumed to be Stein, then the assumption that $E$ is a $(n-3)$-complete compact set can be relaxed as follows: $E$ is closed (not necessarily compact) and there is a countable family $\left\{V_{\nu}\right\}_{\nu=1}^{\infty}$ of $(n-3)$-complete open neighborhoods of $E$ with

$$
V_{\nu} \supset V_{\nu+1} \text { for every } \nu \text {, and } \bigcap_{\nu=1}^{\infty} V_{\nu}=E \text {. }
$$

As a matter of fact, given a $C^{\infty}$ strongly plurisubharmonic exhaustion function $p: M \rightarrow \mathbf{R}$, with $p \leq 0$ on $K$, the set $E^{\prime}=E \cap\{p \leq 0\}$ verifies the assumption of Corollary 2.2, since the open sets $U_{\nu}=V_{\nu} \cap\{p<1 / \nu\}, \nu=1,2, \ldots$, are $(n-3)$-complete (cf. [21]) and make a neighborhood basis of $E^{\prime}$.

Corollary 2.2, in view of the above observation too, improves some results of [16 and 17].

Now we discuss another consequence of Theorem 2.1, which is indeed the main result of this section and will be applied afterwards. Preliminarily let us give the following

Definition. A compact set $E \subset M$ is called q-convex $(0 \leq q \leq n)$ in case there exists a sequence $\left\{u_{\nu}\right\}_{\nu=1}^{\infty}$ of $C^{\infty}$ strongly q-plurisubharmonic exhaustion functions on $M$ such that

$$
\left\{u_{\nu}<0\right\} \ni\left\{u_{\nu+1}<0\right\} \text { for every } \nu, \quad \text { and } \bigcap_{\nu=1}^{\infty}\left\{u_{\nu}<0\right\}=E \text {. }
$$

Clearly, the $q$-convexity of $E$ implies the $q$-completeness of both $M$ and E.

This notion of $q$-convexity generalizes in a quite natural way to the case of a $q$-complete manifold the standard notion of holomorphic convexity in a Stein manifold. In fact, if $M$ is Stein (i.e. 0-complete), then it is known that the holomorphic convexity of a compact set $E \subset M$ amounts to the existence of a sequence $\left\{u_{\nu}\right\}$ as above, with each $u_{\nu}$ being strongly plurisubharmonic (cf. [10, Theorem 5.1.6]). On the other hand, even in a Stein manifold a $q$-convex compact set need not be holomorphically convex, unless $q=0$.

That being stated, we can prove

Theorem 2.3. Suppose that $M$ is $(n-2)$-complete and that there is a $(n-2)$ convex compact set $E \subset M$ with $E \cap \bar{D}=K$. Then, for any $W$, the global extension property holds for $\operatorname{CR}(b D \backslash K, W)$.

Proof. If $\left\{u_{\nu}\right\}$ is a sequence as described above, which exhibits the $(n-2)$ convexity of $E$, let us set

$$
U_{\nu}=\left\{u_{\nu}<0\right\}, \quad \nu=1,2, \ldots
$$


Then, in view of the discussion after the proof of Theorem 2.1, all we have to prove is that, for every $\nu$, the homomorphism

$$
H_{c}^{2}\left(U_{\nu}, \mathscr{O}(W)\right) \rightarrow H_{c}^{2}(M, \mathscr{O}(W))
$$

induced by inclusion is injective, for any $W$.

Consider the spaces $H^{n-2}\left(M, \Omega^{n}\left(W^{*}\right)\right), H^{n-2}\left(U_{\nu}, \Omega^{n}\left(W^{*}\right)\right)$, with their natural topologies obtained by means of the Čech cohomology. By a result of Andreotti and Grauert [1, p. 248], the homomorphism

$$
H^{n-2}\left(M, \Omega^{n}\left(W^{*}\right)\right) \rightarrow H^{n-2}\left(U_{\nu}, \Omega^{n}\left(W^{*}\right)\right)
$$

induced by inclusion has a dense image. The same is then true of the induced homomorphism

$$
{ }^{\sigma} H^{n-2}\left(M, \Omega^{n}\left(W^{*}\right)\right) \rightarrow{ }^{\sigma} H^{n-2}\left(U_{\nu}, \Omega^{n}\left(W^{*}\right)\right)
$$

of the associated separated spaces. Hence the transposed homomorphism

$$
\text { Hom cont }\left({ }^{\sigma} H^{n-2}\left(U_{\nu}, \Omega^{n}\left(W^{*}\right)\right), \mathbf{C}\right) \rightarrow \operatorname{Hom} \operatorname{cont}\left({ }^{\sigma} H^{n-2}\left(M, \Omega^{n}\left(W^{*}\right)\right), \mathbf{C}\right)
$$

is injective.

On the other hand, both $M$ and $U_{\nu}$ are $(n-2)$-complete (cf. [21]) for the $(n-2)$-completeness of $\left.U_{\nu}\right)$, and it is known that consequently the spaces $H_{c}^{2}(M, \mathscr{O}(W))$ and $H_{c}^{2}\left(U_{\nu}, \mathscr{O}(W)\right)$ are separated (cf. [2]). It follows, by the Serre duality, that there is a commutative diagram

$$
\begin{aligned}
H_{c}^{2}\left(U_{\nu}, \mathscr{O}(W)\right) & \rightarrow H_{c}^{2}(M, \mathscr{O}(W)) \\
\uparrow \cong & \cong \uparrow
\end{aligned}
$$

Hom cont $\left({ }^{\sigma} H^{n-2}\left(U_{\nu}, \Omega^{n}\left(W^{*}\right)\right), \mathbf{C}\right) \rightarrow$ Hom $\operatorname{cont}\left({ }^{\sigma} H^{n-2}\left(M, \Omega^{n}\left(W^{*}\right)\right), \mathbf{C}\right)$ and hence we get the desired conclusion. Q.E.D.

Note that the assumptions of Theorem 2.3 hold in particular in case $M$ is Stein and $\hat{K}_{M} \cap \bar{D}=K$ (with $\hat{K}_{M}$ being the $\mathscr{O}(M)$-hull of $K$ ), and in fact they amount to saying that this is the case for $n=2$. In this situation the global extension property from $b D \backslash K$ has already been proved in [11] by different techniques for the case of continuous CR-functions.

We conclude this section by deriving a corollary of Theorem 2.3 which generalizes widely what was proved in [13].

Corollary 2.4. Suppose that $M$ is q-complete, $0 \leq q \leq n-2$, and that there is $a C^{\infty}(n-2-q)$-plurisubharmonic function $\rho: M \rightarrow \mathbf{R}$ such that

$$
K \subset\{\rho=0\}, \quad \bar{D} \backslash K \subset\{\rho>0\} .
$$

Then, for any $W$, the global extension property holds for $\operatorname{CR}(b D \backslash K, W)$.

Proof. Let $p$ be a $C^{\infty}$ strongly $q$-plurisubharmonic exhaustion function on $M$ such that $K \subset\{p<0\}$. One can easily verify that, if $\varepsilon$ is any positive real number, the function $e^{\rho}-1+\varepsilon p$ is a strongly $(n-2)$-plurisubharmonic exhaustion function on $M$; therefore the set

$$
E_{\varepsilon}=\left\{e^{\rho}-1+\varepsilon p \leq 0\right\}
$$


is compact and $(n-2)$-convex in $M$. Moreover, by our assumptions, it is plain that $E_{\varepsilon} \cap \bar{D}$ is contained in any arbitrarily small neighborhood of $K$, provided $\varepsilon$ is small enough.

Then let us set

$$
D_{\varepsilon}=D \backslash E_{\varepsilon}, \quad K_{\varepsilon}=\bar{D} \cap b E_{\varepsilon} .
$$

Thus, for all sufficiently small $\varepsilon, D_{\varepsilon}$ is a domain and $b D_{\varepsilon} \backslash K_{\varepsilon}=b D \backslash E_{\varepsilon}$ is connected, since so are $D$ and $b D \backslash K$, respectively. Therefore, by resorting to Theorem 2.3, we infer that the global extension property holds for $\operatorname{CR}\left(b D_{\varepsilon} \backslash K_{\varepsilon}, W\right)$ for all sufficiently small $\varepsilon$.

The conclusion is then a consequence of the uniqueness of the extension of every CR-object. Q.E.D.

\section{THE ONE SIDE EXTENSION PROPERTY}

The last results of the previous section can be applied to the question of the one side extension property for the CR-objects defined on a piece of a real hypersurface of $\mathbf{C}^{n} \quad(n \geq 2)$.

Let us consider a relatively closed real hypersurface $S$ of an open set $\Omega \Subset \mathbf{C}^{n}$. $S$ is assumed to be connected, smooth of class $C^{v} \quad(1 \leq v \leq \infty)$ or of class $C^{\omega}$ and orientable; instead no regularity assumptions are made on its topological boundary $\dot{S}=\operatorname{Clos}_{\mathrm{C}^{n}}(S) \backslash S$.

Given a holomorphic complex vector bundle $W$ on $\mathbf{C}^{n}$, according to the previous sections we let $\operatorname{CR}(S, W)$ denote the set of the CR-objects with coefficients in $W$ defined on $S$. Of course, in the present setting, as there exist global holomorphic trivializations of $W$, there is no loss of generality in thinking of $W$ just as a complex vector space.

By saying that the one side extension property holds for $\mathrm{CR}(S, W)$ we shall mean the following: There exists an open neighborhood $U \subset \Omega$ of $S$ such that $U \backslash S$ has two connected components $U^{+}, U^{-}$and every $f \in \operatorname{CR}(S, W)$ is the boundary values, in the appropriate sense, of a unique holomorphic section $F \in \Gamma\left(U^{ \pm}, \mathscr{O}(W)\right)$; moreover, if $f \in C^{u}(S, W), 0 \leq u \leq v$, then $F$ admits an extension of class $C^{u}$ to $U^{ \pm} \cup S$ which coincides with $f$ on $S$.

In the first place let us prove

Theorem 3.1. Suppose there is a $(n-2)$-convex compact set $E \subset \mathbf{C}^{n}$ such that

$$
\dot{S} \subset E, \quad S \subset \mathbf{C}^{n} \backslash E .
$$

Then, for any $W$, the one side extension property holds for $\operatorname{CR}(S, W)$.

Proof. We will prove that there is an open domain $D \Subset \mathbf{C}^{n}$ with

$$
D \subset \mathbf{C}^{n} \backslash E, \quad S \subset b D \quad \text { and } \quad b D \backslash S \subset E .
$$

After that the thesis will be a straightforward consequence of Theorem 2.3, for $K=b D \backslash S$.

First of all we need to prove that

$$
H^{2 n-2}(E, \mathbf{C})=0 .
$$


With the notation as in the proof of Theorem 2.3, we have

$$
H^{2 n-2}(E, \mathbf{C}) \cong \lim _{\longrightarrow} H^{2 n-2}\left(U_{\nu}, \mathbf{C}\right),
$$

and hence (1) will follow if we show that, for every $\nu, H^{2 n-2}\left(U_{\nu}, \mathbf{C}\right)$ vanishes. Let us consider first the case $n=2$ : in this case $U_{\nu}$ is a Runge open set in $\mathbf{C}^{2}$, and then the vanishing of $H^{2}\left(U_{\nu}, \mathbf{C}\right)$ follows from a known result on Runge domains in $\mathbf{C}^{n}$ (cf. [10, p. 58]). Next, assume that $n \geq 3$, and consider the following subcomplex $\mathscr{\mathscr { E }}^{* *}\left(U_{\nu}\right)$ of the complex $\mathscr{E}^{* *}\left(U_{\nu}\right)$ of $C^{\infty}$ complexvalued differential forms on $U_{\nu}$ :

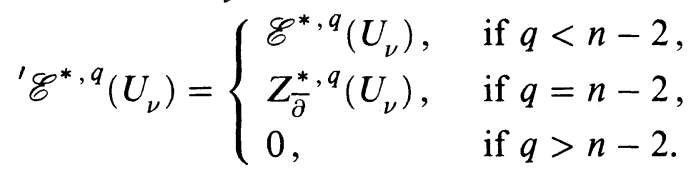

Since $U_{\nu}$ is $(n-2)$-complete, a standard spectral sequences argument shows that the inclusion ${ }^{\prime} \mathscr{E}^{* *}\left(U_{\nu}\right) \subset \mathscr{E}^{* *}\left(U_{\nu}\right)$ induces an isomorphism on total cohomology. This implies in particular, on account of the Dolbeault and the De Rham isomorphisms, that there is a surjective continuous homomorphism

$$
H^{n-2}\left(U_{\nu}, \Omega^{n}\right) \rightarrow H^{2 n-2}\left(U_{\nu}, \mathbf{C}\right) .
$$

On the other hand, since the homomorphism $H^{n-2}\left(\mathbf{C}^{n}, \Omega^{n}\right) \rightarrow H^{n-2}\left(U_{\nu}, \Omega^{n}\right)$ induced by inclusion has a dense image (cf. the proof of Theorem 2.3), ${ }^{\sigma} H^{n-2}\left(\dot{U}_{\nu}, \Omega^{n}\right)$ vanishes. Hence $H^{2 n-2}\left(U_{\nu}, \mathbf{C}\right)={ }^{\sigma} H^{2 n-2}\left(U_{\nu}, \mathbf{C}\right)$ vanishes as well. This concludes the proof of $(1)$.

Now, (1) implies, via the cohomology sequence with compact supports

$$
\cdots \rightarrow H^{2 n-2}(E, \mathbf{C}) \rightarrow H_{c}^{2 n-1}\left(\mathbf{C}^{n} \backslash E, \mathbf{C}\right) \rightarrow H_{c}^{2 n-1}\left(\mathbf{C}^{n}, \mathbf{C}\right) \rightarrow \cdots,
$$

that

$$
H_{c}^{2 n-1}\left(\mathbf{C}^{n} \backslash E, \mathbf{C}\right)=0 .
$$

Consider then the cohomology sequence with compact supports

$$
\begin{aligned}
\cdots \rightarrow H_{c}^{2 n-1}\left(\mathbf{C}^{n} \backslash E, \mathbf{C}\right) & \rightarrow H_{c}^{2 n-1}(S, \mathbf{C}) \\
& \rightarrow H_{c}^{2 n}\left(\left(\mathbf{C}^{n} \backslash E\right) \backslash S, \mathbf{C}\right) \rightarrow H_{c}^{2 n}\left(\mathbf{C}^{n} \backslash E, \mathbf{C}\right) \rightarrow 0 .
\end{aligned}
$$

Here we have $H_{c}^{2 n}\left(\mathbf{C}^{n} \backslash E, \mathbf{C}\right) \cong \mathbf{C}$, because $E$ does not disconnect $\mathbf{C}^{n}$ (see the discussion after Theorem 2.1), and $H_{c}^{2 n-1}(S, \mathbf{C}) \cong \mathbf{C}$, because $S$ is connected and orientable. Therefore, due to (2), we get

$$
H_{c}^{2 n}\left(\left(\mathbf{C}^{n} \backslash E\right) \backslash S, \mathbf{C}\right) \cong \mathbf{C} \oplus \mathbf{C} .
$$

Finally, (3) means that $\left(\mathbf{C}^{n} \backslash E\right) \backslash S$ is made by two connected components. Clearly, by the compactness of $E \cup S$, one of these components must be relatively compact. This proves the existence of $D$. Q.E.D.

In the same way as we derived Corollary 2.4 from Theorem 2.3, now we can derive from Theorem 3.1 the following other result relating to the one side extension property. 
Corollary 3.2. Suppose that there is a $C^{\infty}(n-2)$-plurisubharmonic function $\rho$ on $\mathbf{C}^{n}$ such that

$$
\dot{S} \subset\{\rho=0\}, \quad S \subset\{\rho>0\} .
$$

Then, for any $W$, the one side extension property holds for $\operatorname{CR}(S, W)$.

As the proof is essentially a repetition of that of Corollary 2.4 , we dispense with it.

It is worth while to point out that Corollary 3.2 implies the local extension theorems of Hans Lewy's type for CR-functions, CR-distributions and CR-hyperfunctions. Indeed we have

Proposition 3.3. Let $\Sigma$ be a piece of a $C^{2}$ regular hypersurface in $\mathbf{C}^{n}$, and let $z^{0} \in \Sigma$ be a point where the Levi form is not degenerate. Then we can find an open neighborhood $\Omega \Subset \mathbf{C}^{n}$ of $z^{0}$ in such a way that $S=\Sigma \cap \Omega$ is a relatively closed connected hypersurface in $\Omega$ and the assumptions of Corollary 3.2 are fulfilled.

Proof. Arguing as in [10, p. 51], we may assume that $\Sigma$ is a relatively closed hypersurface in an open neighborhood $\omega$ of the origin $O$, that $z^{0}=O$ and that $\Sigma$ is represented in $\omega$ by an equation $r\left(z_{1}, \ldots, z_{n}\right)=0$, with $r$ being of the form

$$
r \equiv \operatorname{Im}\left(z_{2}\right)+\sum_{j, k}^{1, n} A_{j k} z_{j} \bar{z}_{k}+O\left(|z|^{3}\right),
$$

and $A_{11}>0$.

We claim that there is no loss of generality in assuming further that the binary Hermitian form $\sum_{j, k}^{1,2} A_{j k} z_{j} \bar{z}_{k}$ is positive definite. As a matter of fact, let $s=\exp (c r)-1$, which clearly is another defining function of $\Sigma$ for any constant $c>0$; then, since

$$
s \equiv c\left(\operatorname{Im}\left(z_{2}\right)+\sum_{j, k}^{1, n} A_{j k} z_{j} \bar{z}_{k}+\frac{c}{4}\left|z_{2}\right|^{2}+O\left(|z|^{3}\right)\right),
$$

after replacing $r$ by $s / c$ for $c$ large enough, we have a defining function of $\Sigma$ with the asserted features.

Now, since

$$
r\left(z_{1}, z_{2}, 0, \ldots, 0\right) \equiv \operatorname{Im}\left(z_{2}\right)+\sum_{j, k}^{1,2} A_{j k} z_{j} \bar{z}_{k}+O\left(\left|\left(z_{1}, z_{2}, 0, \ldots, 0\right)\right|^{3}\right)
$$

and $\sum_{j, k}^{1,2} A_{j k} z_{j} \bar{z}_{k}$ is positive definite, we can choose two small real numbers $\delta>0, \varepsilon>0$ so that

$$
\Omega=\left\{z \in \mathbf{C}^{n}:\left|z_{1}\right|^{2}+\left|z_{2}\right|^{2}<\delta,-\operatorname{Im}\left(z_{2}\right)+\left|z_{3}\right|^{2}+\cdots+\left|z_{n}\right|^{2}<\varepsilon\right\} \subset \omega,
$$


$r>0$ on the part of $b \Omega$ where $\left|z_{1}\right|^{2}+\left|z_{2}\right|^{2}=\delta$ and $S=\Sigma \cap \Omega$ is connected and relatively closed in $\Omega$. Therefore we have:

$$
\begin{aligned}
\dot{S}= & \Sigma \cap b \Omega \subset\left\{z \in \mathbf{C}^{n}:-\operatorname{Im}\left(z_{2}\right)+\left|z_{3}\right|^{2}+\cdots+\left|z_{n}\right|^{2}=\varepsilon\right\}, \\
& S \subset\left\{z \in \mathbf{C}^{n}:-\operatorname{Im}\left(z_{2}\right)+\left|z_{3}\right|^{2}+\cdots+\left|z_{n}\right|^{2}<\varepsilon\right\} .
\end{aligned}
$$

Then, since the function $\rho=\varepsilon+\operatorname{Im}\left(z_{2}\right)-\left|z_{3}\right|^{2}-\cdots-\left|z_{n}\right|^{2}$ is a real-analytic $(n-2)$-plurisubharmonic function on $\mathbf{C}^{n}$, we conclude that $S$ verifies the assumptions of Corollary 3.2. Q.E.D.

Thus Corollary 3.2 does imply the local extension theorems of Hans Lewy's type. On the other hand it trivially implies, for $\dot{S}=\varnothing$ and via the Hartogs theorem, the corresponding global extension theorems for the CR-objects defined on the boundary of a bounded domain of $\mathbf{C}^{n}$. Therefore Corollary 3.2 can be considered as a proposition providing a unification of the local and global extension theorems in $\mathbf{C}^{n}$. Another such unification, based on different ideas, has already been obtained previously by Fichera [7].

Actually we wonder if Corollary 3.2 could imply also a recent theorem of Trépreau [24] to the effect that the local one side extension property holds at a point $z^{0} \in \Sigma$, provided there are no germs of complex hypersurfaces passing through $z^{0}$ and contained in $\Sigma$. One should prove that the thesis of Proposition 3.3 is still true under this weaker assumption; however the matter seems to be rather complicated and we limit ourselves to notice that, at least in the case when $n=2$ and $\Sigma$ is smooth of class $C^{\infty}$, a proof could probably be developed along the same lines as in [5, Theorem 3.1.10].

\section{Global extension on unbounded domains of $\mathbf{C}^{n}$}

In this last section we discuss the following question.

Given an unbounded domain $D \subset \mathbf{C}^{n}, n \geq 2$, with boundary $b D$ connected and smooth of class $C^{v}, 1 \leq v \leq \infty$, or of class $C^{\omega}$, under which conditions does the global extension property hold for the CR-objects defined on $b D$ ?

We have already considered this question in [15] for the case of continuous CR-functions; here, in view of the previous results of this paper, we are able to prove a quite more general theorem than that of [15].

Let us consider the hyperplane "at infinity" $\mathbf{P}_{\infty}^{n-1}$ of $\mathbf{C}^{n}$, so that

$$
\mathbf{C}^{n}=\mathbf{P}^{n} \backslash \mathbf{P}_{\infty}^{n-1}
$$

For every subset $G \subset \mathbf{C}^{n}$, let us set

$$
\widetilde{G}=\operatorname{Clos}_{\mathbf{P}^{n}}(G) ;
$$

thus $\widetilde{G}$ is obtained by adding to $\bar{G}=\operatorname{Clos}_{\mathrm{C}^{n}}(G)$ its points at infinity.

With $D$ being as above, we have

Theorem 4.1. Suppose there exist $q$ entire analytic hypersurfaces $H_{1}, \ldots, H_{q}$ of $\mathbf{C}^{n}, 1 \leq q \leq n-1$, so that

$$
\widetilde{D} \cap \widetilde{H}_{1} \cap \cdots \cap \widetilde{H}_{q}=\varnothing .
$$


Then, if $W$ is any holomorphic complex vector bundle of finite rank on $\mathbf{P}^{n}$, the global extension property holds for $\mathrm{CR}(b D, W)$.

Proof. In the first place we observe that, since for an entire hypersurface $H \subset$ $\mathbf{C}^{n}$ which is not algebraic one has $\widetilde{H} \supset \mathbf{P}_{\infty}^{n-1}$ (cf. [20]), the condition of the theorem enforces the algebraicity of at least one of the hypersurfaces $H_{1}, \ldots, H_{q}$; moreover, if $H_{1}, \ldots, H_{q^{\prime}}, 1 \leq q^{\prime} \leq q$, are all the algebraic ones, for the same reason we have

$$
\left(\widetilde{D} \cap \widetilde{H}_{1} \cap \cdots \cap \widetilde{H}_{q^{\prime}}\right) \cap \mathbf{P}_{\infty}^{n-1}=\varnothing,
$$

which means that $\widetilde{D} \cap \widetilde{H}_{1} \cap \cdots \cap \widetilde{H}_{q^{\prime}}$ is a compact subset of $\mathbf{C}^{n}$. Therefore, if $q^{\prime}<n-1$, we can obviously choose $n-1-q^{\prime}$ further algebraic hypersurfaces $H_{q^{\prime}+1}^{\prime}, \ldots, H_{n-1}^{\prime}$, such that

$$
\widetilde{D} \cap \widetilde{H}_{1} \cap \cdots \cap \widetilde{H}_{q^{\prime}} \cap \widetilde{H}_{q^{\prime}+1}^{\prime} \cap \cdots \cap \widetilde{H}_{n-1}^{\prime}=\varnothing .
$$

Hence we may assume from the beginning that $q=n-1$ and $H_{1}, \ldots, H_{n-1}$ are all algebraic.

Now, let us set

$$
M=\mathbf{P}^{n} \backslash\left(\widetilde{H}_{1} \cap \cdots \cap \widetilde{H}_{n-1}\right), \quad K=\widetilde{D} \cap \mathbf{P}_{\infty}^{n-1} .
$$

Clearly $D \Subset M$ and the boundary of $D$ in $M$ is made by $b D=\operatorname{Bd}_{\mathbf{C}^{n}}(D)$ and by $K$.

We will construct a sequence $\left\{u_{\nu}\right\}$ of $C^{\infty}$ strongly $(n-2)$-plurisubharmonic exhaustion functions on $M$ such that

(*) $\quad\left\{u_{\nu}<0\right\} \ni\left\{u_{\nu+1}<0\right\}$ for all $\nu, \quad$ and $\widetilde{D} \cap \bigcap_{\nu=1}^{\infty}\left\{u_{\nu}<0\right\}=K$;

the conclusion will then follow from Theorem 2.3 for $E=\bigcap_{\nu=1}^{\infty}\left\{u_{\nu}<0\right\}$.

Let $\left(\zeta_{0}, \ldots, \zeta_{n}\right)$ be homogeneous coordinates in $\mathbf{P}^{n}$ with $\mathbf{P}_{\infty}^{n-1}=\left\{\zeta_{0}=0\right\}$ and let

$$
F_{1}\left(\zeta_{0}, \ldots, \zeta_{n}\right)=0, \ldots, F_{n-1}\left(\zeta_{0}, \ldots, \zeta_{n}\right)=0
$$

be equations of $\widetilde{H}_{1}, \ldots, \widetilde{H}_{n-1}$, respectively. After taking suitable powers, we may assume that $F_{1}, \ldots, F_{n-1}$ have the same degree, $r$ say.

Consider the two real-analytic functions $\rho, p: M \rightarrow \mathbf{R}$ defined by

$$
\begin{gathered}
{\left[\zeta_{0}, \ldots, \zeta_{n}\right] \stackrel{\rho}{\mapsto} \frac{\left|\zeta_{0}^{r}\right|^{2}}{\left|F_{1}\right|^{2}+\cdots+\left|F_{n-1}\right|^{2}},} \\
{\left[\zeta_{0}, \ldots, \zeta_{n}\right] \stackrel{p}{\mapsto} \log \frac{\left(\left|\zeta_{0}\right|^{2}+\cdots+\left|\zeta_{n}\right|^{2}\right)^{r}}{\left|F_{1}\right|^{2}+\cdots+\left|F_{n-1}\right|^{2}}+C,}
\end{gathered}
$$

with $C$ being a real constant such that $K \subset\{p<0\}$.

Then set, for every positive integer $\nu$,

$$
u_{\nu}=\rho+(1 / \nu) p .
$$


We claim that these functions have the asserted properties. As a matter of fact, let $M_{j}=M \backslash\left\{F_{j}=0\right\} ; j=1, \ldots, n-1$, and consider the holomorphic maps $f_{j}: M_{j} \rightarrow \mathbf{C}^{n-1}$ given by

$$
\left[\zeta_{0}, \ldots, \zeta_{n}\right] \stackrel{f_{j}}{\mapsto}\left(\zeta_{0}^{r} / F_{j}, F_{1} / F_{j}, \ldots, F_{j-1} / F_{j}, F_{j+1} / F_{j}, \ldots, F_{n-1} / F_{j}\right),
$$

$j=1, \ldots, n-1$. Then on $M_{j}$ we have

$$
\begin{aligned}
\partial \bar{\partial} u_{\nu}= & f_{j}^{*} \partial \bar{\partial}\left(\frac{\left|z_{1}\right|^{2}}{1+\left|z_{2}\right|^{2}+\cdots+\left|z_{n-1}\right|^{2}}+\frac{1}{\nu} \log \frac{1}{1+\left|z_{2}\right|^{2}+\cdots+\left|z_{n-1}\right|^{2}}\right) \\
& +\frac{1}{\nu} \partial \bar{\partial} \log \frac{\left(\left|\zeta_{0}\right|^{2}+\cdots+\left|\zeta_{n}\right|^{2}\right)^{r}}{\left|F_{j}\right|^{2}}
\end{aligned}
$$

hence we see that $u_{\nu}$ is strongly $(n-2)$-plurisubharmonic on $M_{j}$ : so it is then on all of $M$, since $M=\bigcup_{j=1}^{n-1} M_{j}$. Moreover $u_{\nu}$ is exhaustive, since so is $p$ and $\rho$ is bounded from below. Finally, the sequence $\left\{u_{\nu}\right\}$ clearly verifies $(*)$. Q.E.D.

We conclude by observing that the assumption of Theorem 4.1, as is apparent from the first part of the proof, can be translated in the following way: There exist $q$ polynomials $P_{1}, \ldots, P_{q} \in \mathbf{C}\left[z_{1}, \ldots, z_{n}\right], 1 \leq q \leq n-1$, of respective degrees $r_{1}, \ldots, r_{q}$, so that

$$
D \subset\left\{z \in \mathbf{C}^{n}:\left|P_{1}(z)\right|^{2 r / r_{1}}+\cdots+\left|P_{q}(z)\right|^{2 r / r_{q}}>\left(1+|z|^{2}\right)^{r}\right\},
$$

with $r$ being the least common multiple of $r_{1}, \ldots, r_{q}$.

Remark added during revision. Recently a paper by B. Jöricke (Removable singularities of CR-functions, Ark. Mat. 26 (1988), 117-143) appeared, which also deals with the global extension property of CR-functions from a part of the boundary of a relatively compact domain $D$.

Jöricke considers the case where $D$ is a $C^{2}$-bounded strongly pseudoconvex domain in $\mathbf{C}^{n}$ and obtains some relevant results in this area by functiontheoretic techniques based on the Kontinuitätssatz.

\section{REFERENCES}

1. A. Andreotti and H. Grauert, Théorèmes de finitude pour la cohomologie des espaces complexes, Bull. Soc. Math. France 90 (1962), 193-259.

2. A. Andreotti and A. Kas, Duality on complex spaces, Ann. Scuola Norm. Sup. Pisa $\mathbf{1 6}$ (1973), 187-263.

3. C. Bănică and O. Stănăşilă, Algebraic methods in the global theory of complex spaces, Wiley, London and New York, 1976.

4. G. E. Bredon, Sheaf theory, McGraw-Hill, New York, 1967.

5. D. Catlin, Boundary behaviour of holomorphic functions on pseudoconvex domains, Dissertation, Princeton University, 1978.

6. A. Dold, Lectures on algebraic topology, Springer-Verlag, Berlin, Heidelberg and New York, 1972. 
7. G. Fichera, Unification of global and local existence theorems for holomorphic functions of several complex variables, Atti Accad. Naz. Lincei Mem. Cl. Sci. Fis. Mat. Natur. Sez. Ia (8) 18 (1986), 61-83.

8. R. Godement, Topologie algébrique et théorie des faisceaux, Hermann, Paris, 1958.

9. F. R. Harvey and H. B. Lawson, On boundaries of complex analytic varieties. I, Ann. of Math. (2) 102 (1975), 223-290.

10. L. Hörmander, An introduction to complex analysis in several variables, North-Holland, Amsterdam, 1973.

11. C. Laurent-Thiébaut, Sur l'extension des fonctions $C R$ dans une variété de Stein, Ann. Mat. Pura Appl. (4) 150 (1988), 141-151.

12. S. Lojasiewicz and G. Tomassini, Valeurs au bord des formes holomorphes, Several Complex Variables (Proc. Internat. Conf., Cortona, Italy, 1976-1977), Scuola Norm. Sup., Pisa, 1978, pp. 222-245.

13. G. Lupacciolu and G. Tomassini, Un teorema di estensione per le CR-funzioni, Ann. Mat. Pura Appl. (4) 137 (1984), 257-263.

14. G. Lupacciolu, A theorem on holomorphic extension of CR-functions, Pacific J. Math. 124 (1986), 177-191.

15. __ Valeurs au bord de fonctions holomorphes dans des domaines non bornés de $\mathbf{C}^{n}, \mathrm{C}$. R. Acad. Sci. Paris Sér. I Math. 304 (1987), 67-69.

16. __ Holomorphic continuation in several complex variables, Pacific J. Math. 128 (1987), 117-126.

17. A. Perotti, Extension of CR-forms and related problems, Rend. Sem. Mat. Univ. Padova 77 (1987), 37-55.

18. J. C. Polking and R. O. Wells, Jr., Boundary values of Dolbeault cohomology classes and a generalized Bochner-Hartogs Theorem, Abh. Math. Sem. Univ. Hamburg 47 (1978), 3-24.

19. J. P. Serre, Un théorème de dualité, Comment. Math. Helv. 29 (1955), 9-26.

20. B. V. Shabat, Distribution of values of holomorphic mappings, Transl. Math. Mono., no. 61, Amer. Math. Soc., Providence, R.I., 1985.

21. G. Sorani and V. Villani, q-complete spaces and cohomology, Trans. Amer. Math. Soc. 125 (1966), 432-448.

22. E. L. Stout, Analytic continuation and boundary continuity of functions of several complex variables, Proc. Edinburgh Roy. Soc. A89 (1981), 63-74.

23. G. Tomassini, Extension of CR-functions, Lecture Notes in Math., vol. 1165, SpringerVerlag, New York, 1986, pp. 294-301.

24. J. M. Trépreau, Sur le prolongement holomorphe des fonctions $C-R$ définies sur une hypersurface réelle de classe $C^{2}$ dans $\mathbf{C}^{n}$, Invent. Math. 83 (1986), 583-592.

Dipartimento di Matematica Istituto "G. Castelnuovo", Universitá degli Studi di Roma "La Sapienza", Piazzale Aldo Moro, 2, I-00185 Roma, Italy 